\title{
Methods and algorithms for control input placement in complex networks
}

Gustav Lindmark

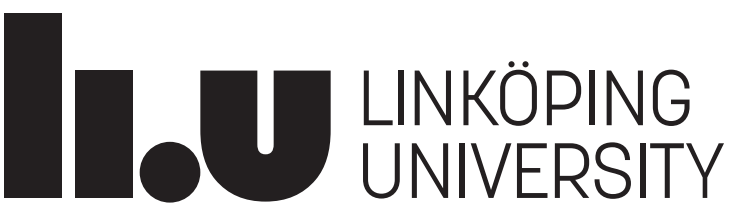


This is a Swedish Licentiate's Thesis.

Swedish postgraduate education leads to a Doctor's degree and/or a Licentiate's degree.

A Doctor's Degree comprises 240 ECTS credits (4 years of full-time studies).

A Licentiate's degree comprises 120 ECTS credits, of which at least 60 ECTS credits constitute a Licentiate's thesis.

Linköping studies in science and technology. Thesis

No. 1814

Methods and algorithms for control input placement in complex networks

Gustav Lindmark

\author{
gustav.lindmarkeliu.se \\ www. control.isy.liu.se \\ Department of Electrical Engineering \\ Linköping University \\ SE-581 83 Linköping \\ Sweden
}

ISBN 978-91-7685-243-9 ISSN 0280-7971

Copyright (C) 2018 Gustav Lindmark

Printed by LiU-Tryck, Linköping, Sweden 2018 
To my family 



\section{Abstract}

The control-theoretic notion of controllability captures the ability to guide a systems behavior toward a desired state with a suitable choice of inputs. Controllability of complex networks such as traffic networks, gene regulatory networks, power grids etc. brings many opportunities. It could for instance enable improved efficiency in the functioning of a network or lead to that entirely new applicative possibilities emerge. However, when control theory is applied to complex networks like these, several challenges arise. This thesis consider some of these challenges, in particular we investigate how control inputs should be placed in order to render a given network controllable at a minimum cost, taking as cost function either the number of control inputs or the energy that they must exert. We assume that each control input targets only one node (called a driver node) and is either unconstrained or unilateral.

A unilateral control input is one that can assume either positive or negative values but not both. Motivated by the many applications where unilateral controls are common, we reformulate classical controllability results for this particular case into a more computationally-efficient form that enables a large scale analysis. We show that the unilateral controllability problem is to a high degree structural and derive theoretical lower bounds on the minimal number of unilateral control inputs from topological properties of the network, similar to the bounds that exists for the minimal number of unconstrained control inputs. Moreover, an algorithm is developed that constructs a near minimal number of control inputs for a given network. When evaluated on various categories of random networks as well as a number of real-world networks, the algorithm often achieves the theoretical lower bounds.

A network can be controllable in theory but not in practice when completely uneasonable amounts of control energy are required to steer it in some direction. For unconstrained control inputs we show that the control energy depends on the time constants of the modes of the network, and that the closer the eigenvalues are to the imaginary axis of the complex plane, the less energy is required for control. We also investigate the problem of placing driver nodes such that the control energy requirements are minimized (assuming that theoretical controllability is not an issue). For the special case with networks having all purely imaginary eigenvalues, several constructive algorithms for driver node placement are developed. In order to understand what determines the control energy in the general case with arbitrary eigenvalues, we define two centrality measures for the nodes based on energy flow considerations: the first centrality reflects the network impact of a node and the second the ability to control it indirectly. It turns out that whether a node is suitable as driver node or not largely depends on these two qualities. By combining the centralities into node rankings we obtain driver node placements that significantly reduce the control energy requirements and thereby improve the "practical degree of controllability". 



\section{Populärvetenskaplig sammanfattning}

Det reglerteoretiska begreppet styrbarhet avser förmågan att föra ett system mot ett önskat tillstånd med ett lämpligt val av styrsignaler. Styrbarhet av komplexa nätverk såsom trafiknätverk, regulatoriska gennätverk, elnät m.m. kan ge stora vinster i form av exempelvis effektivitetsförbättringar samt möjliggöra helt nya applikationer. Men flera utmaningar uppstår när reglerteori tillämpas på komplexa nätverk som dessa. Denna avhandling handlar om några av dessa utmaningar, i synnerhet undersöks hur styrsignaler ska placeras för att göra ett givet nätverk styrbart till lägsta möjliga kostnad. Som kostnadsfunktion tar vi antingen antalet styrsignaler eller den energi som krävs för styrning. Vi utgår ifrån att varje styrsignal endast verkar på en enda nod och är antingen unilateral eller obegränsad till storlek och tecken.

En styrsignal är unilateral om den kan anta enbart positiva eller enbart negativa värden. Motiverade av de många tillämpningsområden där sådana styrsignaler är vanliga, omformulerar vi klassiska styrbarhetsresultat för detta specialfall till en mer beräkningseffektiv form som möjliggör en storskalig analys av komplexa nätverk. Vi visar att det unilaterala styrbarhetsproblemet i hög grad är strukturellt och härleder teoretiska gränser för det lägsta möjliga antalet styrsignaler som ger styrbarhet utifrån topologiska nätverksegenskaper, gränser liknande de som redan finns etablerade för det lägsta möjliga antalet obegränsade styrsignaler. En algoritm har utvecklats som konstruerar ett nära minimalt antal unilaterala styrsignaler. Algoritmens prestanda verifieras på olika kategorier av slumpmässigt genererade nätverk samt ett antal verkliga nätverk hämtade från olika forskningsområden. Det visar sig att den teoretiska undre gränsen för antalet styrsignaler ofta är tillräcklig och kan uppnås.

Ett nätverk som är styrbart i teorin är dock inte styrbart i praktiken i de fall då orimligt stora energimängder krävs för styrning i någon riktning. Vi visar att energin i styrsignalerna beror på tidskonstanterna hos nätverkets moder: energin som krävs för styrning är lägre då modernas egenvärden ligger närmre den imaginära axeln i det komplexa talplanet. Vidare undersöker vi problemet att placera ett begränsat antal styrsignaler på ett sätt som minimerar styrsignalsenergin. För specialfallet nätverk med helt imaginära egenvärden tar vi fram flera konstruktiva algoritmer för detta ändamål. Det generella fallet är dock svårare. För att förstå vad som påverkar styrsignalsenergin definieras två centralitetsmått för nätverkets noder utifrån energiflödesresonemang. Det första måttet speglar nodens nätverkspåverkan och det andra möjligheten att styra noden indirekt via andra noder. Vid placering av styrsignaler visar sig dessa två egenskaper vara viktiga. Genom att rangordna noderna utifrån kombinationer av dessa mått kommer vi fram till styrsignalsplaceringar som påtagligt minskar mängden energi som behövs för styrning och därmed ökar den "praktiska graden av styrbarhet". 



\section{Acknowledgments}

First I would like to thank my supervisor Claudio Altafini and co-supervisor Anders Helmersson for your guidance and encouragement. You have been great sources of ideas and inspiration, I have learned a lot from you!

I would like to thank Svante Gunnarsson and Martin Enqvist, former resp. present head of the automatic control group. It is much thanks to your great leadership that the work environment is so good. To spend my days reading, learning and developing myself, and even get decently paid for it, is truly a privilege. Also thank you Ninna Stensgård for kindly helping me with administrative tasks.

To all my dear colleagues at the automatic control group, thank you for contributing to the nice and friendly atmosphere. We have shared offices, conference trips, lunches, PhD courses, and many memorable conversations (in particular during the coffee breaks).

I would also like to take the opportunity to thank my family. My father Göte and brother Thomas, always supportive and kind. My mother Christina, often in my thoughts, was inspiring with her genuine interest in just about everything. She would have loved to see the academic environment with talents from all over the world that Linköping University has become. My wife Jenny, thank you for your patient and loving support. You and our children are what's truly important to me.

Linköping, June 2018

Gustav Lindmark 



\section{Contents}

\section{Background}

1 Introduction 3

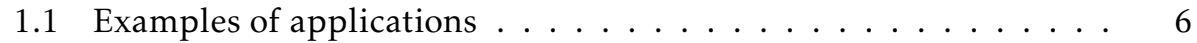

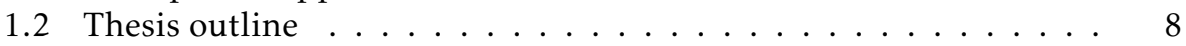

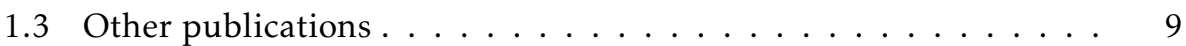

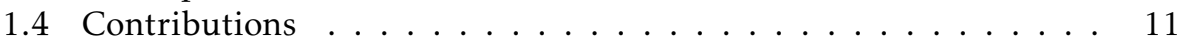

2 Networks and graphs 13

2.1 Graph definitions . . . . . . . . . . . . . . . . . . 13

2.2 Random graphs . . . . . . . . . . . . . . . 15

2.3 Network centrality metrics . . . . . . . . . . . . . . . 17

3 Controllability 19

3.1 Controllability of LTI systems _. . . . . . . . . . . . . . 19

3.2 Controllability with constrained inputs . . . . . . . . . 22

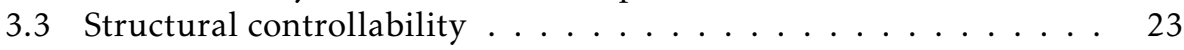

3.4 Minimal controllability of complex networks . . . . . . . . 25

3.5 Minimum energy control of complex networks . . . . . . . 2 26

4 Concluding remarks 29

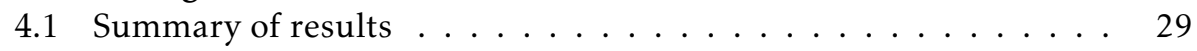

4.2 Future work $\ldots \ldots \ldots \ldots \ldots \ldots \ldots \ldots \ldots \ldots$

$\begin{array}{ll}\text { Bibliography } & 33\end{array}$

\section{Publications}

A Controllability of complex networks with unilateral inputs 39

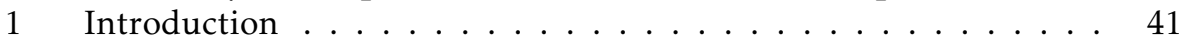

2 Methods ...................... 44 
$3 \quad$ Results ........................ 52

4 Discussion ...................... 60

A Positive linear dependence . . . . . . . . . . . . . 62

B Derivation of conditions for unilateral controllability . . . . . . 63

C Algorithm for construction of unilateral control inputs . . . . . . 64

C.1 Greedy input selection with simple real eigenvectors . . . . 65

C.2 Extension for multidimensional real eigenspaces . . . . . 66

D Derivation of bound on the minimum number of unilateral controls 67

D.1 Structure of the left null space of A . . . . . . . . . 67

D.2 Positive spanning with structurally disjoint vectors . . . . 68

E Derivations of results for specific network structures . . . . . 70

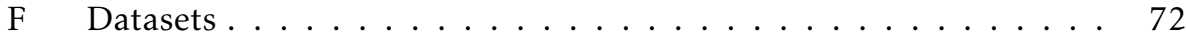

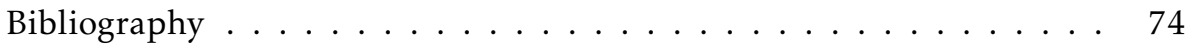

B Minimum energy control for complex networks $\quad 79$

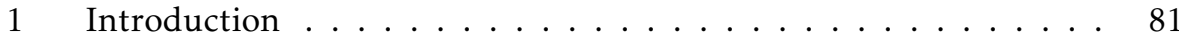

2 Methods ...................... 83

3 Results ....................... 86

4 Discussion ........................ 104

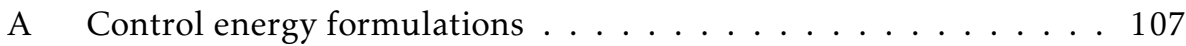

A.1 Finite time horizon . . . . . . . . . . . . . 107

A.2 Infinite time horizon . . . . . . . . . . 108

A.3 Mixed Gramian in finite time horizon . . . . . . . 110

B Control of coupled harmonic oscillators . . . . . . . . . 110

C Controllability with bounded controls . . . . . . . . . . 115

D Datasets . . . . . . . . . . . . . . . . . . . 115

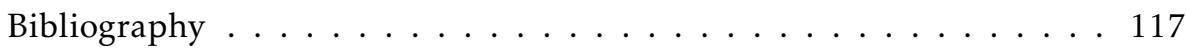

C The role of non-normality for control energy reduction in network $\begin{array}{ll}\text { controllability problems } & 121\end{array}$

1 Introduction . . . . . . . . . . . . . . . 123

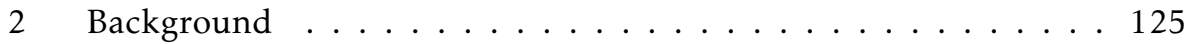

$2.1 \quad$ Notation . . . . . . . . . . . . . . . . . . 125

2.2 Network model . . . . . . . . . . . . . . . . . 126

2.3 Controllability . . . . . . . . . . . . . . 126

3 Driver node placement . . . . . . . . . . . . . . . 127

3.1 Network impact of a driver node . . . . . . . . . . 127

3.2 Minimum energy control of a target node . . . . . . . . 131

3.3 Indirect control of a node . . . . . . . . . . 132

3.4 Ranking the nodes . . . . . . . . . . . . . . 136

3.5 Interpretations of the rankings: non-normality . . . . . 136

3.6 Interpretations of the rankings: flows of energy . . . . . . 137

4 Simulations . . . . . . . . . . . . . . . . . . . 139

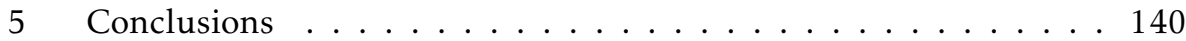

Bibliography . . . . . . . . . . . . . . . 146 
Part I

Background 



\section{1}

\section{Introduction}

In this thesis we consider the problem of controlling large scale, complex networks appearing in a broad spectrum of scientific disciplines, ranging from Biology to Social Sciences, from Technology to Engineering. While control methods have been developed primarily to deal with man-made small-scale technical systems, such as cars, aircraft etc., in many of the contexts in which complex networks are investigated the networks do not represent engineering systems but rather large assemblies of entities (nodes) having some form of interactions represented as the edges of a graph. In this perspective, the control of a network poses challenges which can not always be answered by classical control theory. Formulating and investigating some of these challenges is the scope of this thesis.

Regardless of the specific applicative context, we will always assume that to the nodes of the graph is associated a vector of finite size with state variables, $x(t) \in \mathbb{R}^{n}$. Controlling a complex network means steering the state variables using the available control inputs. For example, the vector element $x_{i}(t)$ can denote the amount of traffic that passes through a node $i$ in a road network, and a control objective could be to avoid congestion. In a food web instead, the state $x_{i}(t)$ can denote the population of a species and a control objective could be to preserve biodiversity. Depending on the context, many are the possible ways to define control inputs on networks, from traffic lights in traffic networks (Tewolde, 2012) to drugs in biological networks (Torres and Altafini, 2016), from dams in irrigation networks (Mareels et al., 2005) to opinion makers in social networks, etc.

Controlling a network does not require to have control authority on each and every node of the network. If the graph of the network is available, then the existing interactions among nodes can be used to propagate the effect of a control input to nodes that are not controlled direct, i.e. the driver nodes can be used to 
indirectly control also the other nodes over the edges of the network. Much of the research in the field concerns how control inputs should be placed in order to render a given network controllable at a minimum cost. If we take as cost function the number of control inputs needed to achieve controllability, then finding a minimal set of driver nodes is a problem of interest. If instead the cost is associated to the energy that the driver nodes must exert in order to steer the state of the network in an arbitrary direction, then another set of driver nodes might be a better choice. In fact, a network that is controllable in a mathematical (control theoretic) sense is often not controllable in practice. It could for instance be the case that completely unreasonable amounts of control energy are required to steer the network in some directions. In the context of large-scale networks, the need to achieve a "practical degree of controllability" is much more pressing than in classical (small-scale) control systems (Bof et al., 2017; Chen et al., 2016; Li et al., 2016; Nacher and Akutsu, 2014; Olshevsky, 2016; Pasqualetti et al., 2014; Summers et al., 2016; Tzoumas et al., 2016; Yan et al., 2012, 2015).

The dynamics of the networks are normally expected to be nonlinear, but poor knowledge of the precise dynamics and the mere complexity of the system make any substantial overarching conclusion difficult to reach without simplifications. For a large scale analysis, a usual starting point in the literature on control of complex networks is instead a Linear Time-Invariant (LTI) network model,

$$
\dot{x}(t)=A x(t)+B u(t) .
$$

The matrix $A \in \mathbb{R}^{n \times n}$, normally sparse, describes the interactions between the entities of the network and the matrix $B \in \mathbb{R}^{n \times m}$ how the control inputs $u(t)=$ $\left[u_{1}(t) \ldots u_{m}(t)\right]^{\top}$ enters into the system. The natural mathematical representation of a network is however a graph, and the network model (1.1) corresponds to the directed graph $\mathcal{G}(A)$ with the set of nodes $\mathcal{V}=\left\{v_{1}, \ldots, v_{n}\right\}$ and edges $\mathcal{E}=$ $\left\{\left(v_{i}, v_{j}\right), i, j\right.$ s.t. $\left.A_{j i} \neq 0\right\}$ with weights given by the numerical values of the elements in $A$. A common assumption is that $B=\left[e_{k_{1}} \ldots e_{k_{m}}\right]$ where $e_{k}, k=1, \ldots, n$, is the $k$-th vector of the canonical basis of $\mathbb{R}^{n}$, which corresponds to placing each control input on a single node of the network called a driver node (the nodes $v_{k_{1}}, \ldots, v_{k_{m}}$ are the driver nodes). Both representations, linear system of ODEs and graphical, are useful and often complement each other.

A graph without edge weights is an entirely topological, or structural, network model. Such model can be used when the interactions between the network components are known but limited or no information is available about their strengths and functional form. Algebraically, this can be represented as each entry of the adjacency matrix $A$ being zero or non-zero. Under some assumptions on the values of the non-zero parameters, this simple model can be used to determine if a system is controllable or not. Such notion is referred to as structural controllability (Lin, 1974) and it is based on the fact that controllability with unrestricted control inputs is a generic property that holds for almost all parameter values. Of course, when the edge weights are known, then a more thorough analysis can be made, which can help in quantifying the practical degree of controllability. 

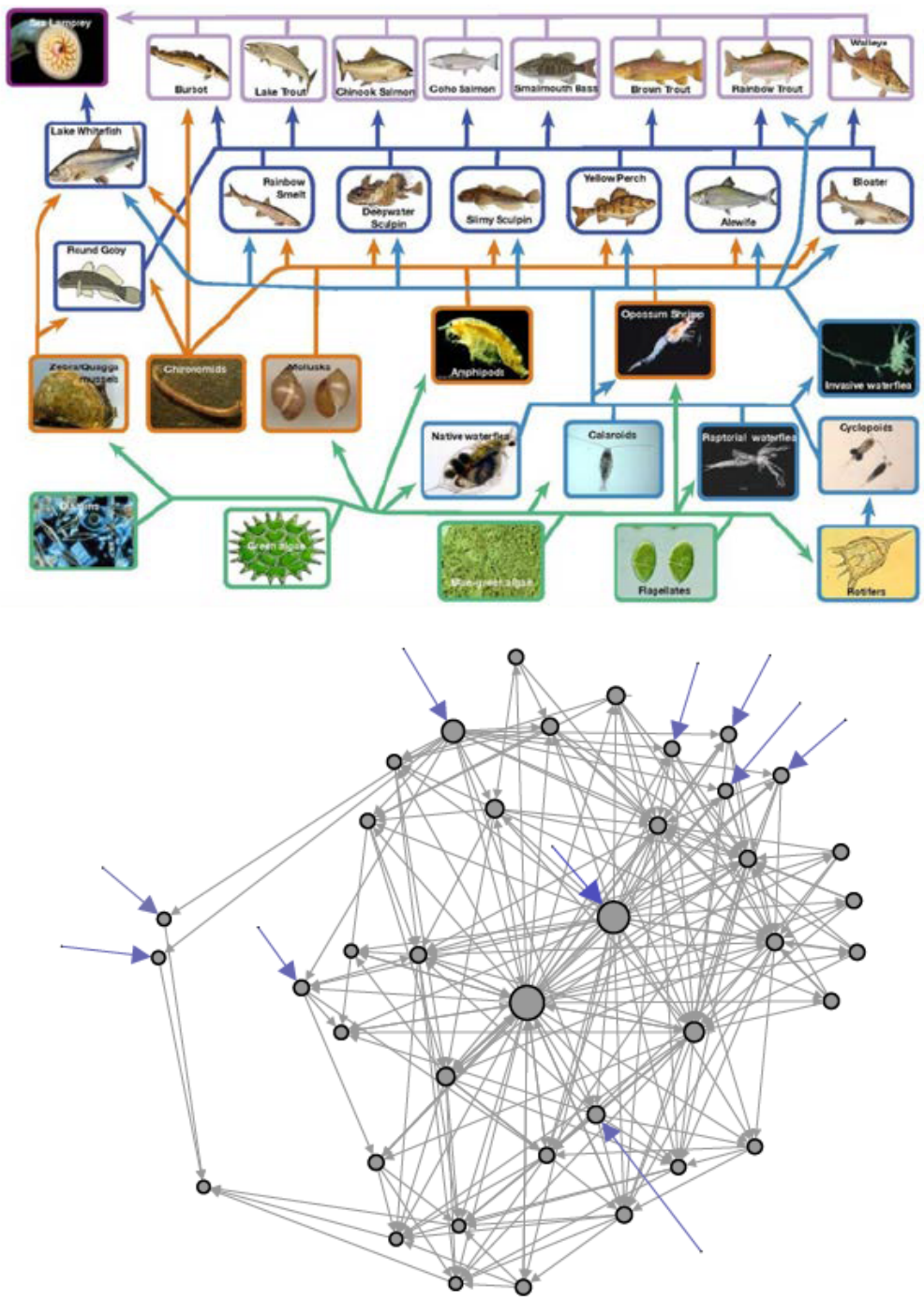

Figure 1.1: Lake Michigan food web (Madenjian et al., 2002). The graph represents a dynamical model in which each state is the population of a species. A minimal set of control inputs that render the network controllable is indicated with blue arrows. For instance feeding and breeding, hunting and fishing are unilateral control inputs in an ecosystem.

Although the different articles in this thesis consider different network models and problem formulations, the overall theme for all of them is investigating how the problem of controlling complex networks can be made more realistic. For instance, in Paper A we restrict the LTI model (1.1) and consider unilateral control inputs, i.e. inputs that can assume either positive or negative values but not both. In many different fields in which controllability of large scale networks 
is studied, the control action is intrinsically constrained, and this is perhaps the most common form of constraint. In Papers $B$ and $C$ we investigate the practical degree of controllability of networks based on energy considerations, and propose methods for driver node placement that significantly reduce the amount of control energy needed to steer the network in different directions.

\subsection{Examples of applications}

Intelligent transportation systems (ITS) are technologies characterized by information, dynamic feedback and automation that address various transportation problems. Perhaps the most important problems are caused by traffic congestion resulting in enormous environmental and economic costs and waste of time. The basis for ITS is the collection and processing of information from sensors integrated in the traffic infrastructure, sensors in the vehicles, satellite information, maps etc. A central system that collects information from different sources can monitor the state of the traffic network, enabling network level traffic control to avoid congestion and achieve optimal utilization of roads and other resources. The actual control inputs can be for instance variable speed limits, control of traffic lights or toll fees. Furthermore, vehicles with telematics already receive information that they benefit from, e.g. real-time route guidance, accident alert systems etc. In a near future with autonomous cars the possibilities for network level traffic control will increase even more.

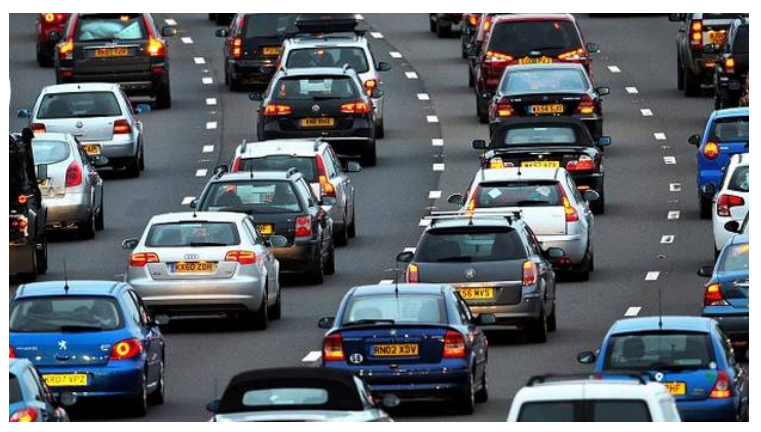

Figure 1.2: New technologies for dynamic traffic network control can reduce the economical and environmental costs of congestion.

The smart power grid refers to technologies that allows for communication between the grid components, sensing along the transmission lines and automation and control for its function. The power grid is a network of generators, transmission lines, substations, transformers, consumers etc. with the task to produce and distribute as much power as is needed. If not successful, the grid voltage could drop, causing the grid to become unstable and in worst case lead to power outage. Traditionally, grid operators have limited information about how the power is flowing through the grid, but with smart grid technologies the state of the network can be monitored and proper control actions taken for its function, leading to increased reliability, availability and efficiency. 
One upcoming challenge for the power grid function is the integration of variable sources of power such as wind power and solar power, which supply an increasing fraction of power to the grid. Since their outputs cannot be controlled there must be other mechanisms for controlling the power supply and demand in the network. One way to do that is to balance the outputs of the variable power sources with other power sources and another is to add energy storage capabilities to the network. Control inputs can also be financial incentives, e.g. variable pricing, for consumers to shift their power demand to off-peak hours.

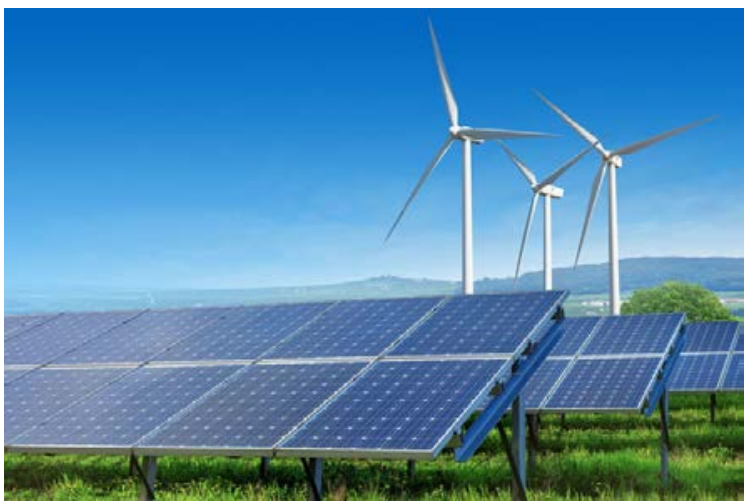

Figure 1.3: The outputs of wind and solar power cannot be controlled, why a smart grid needs other control mechanisms for balancing the power supply and demand as these power sources become increasingly important. 


\subsection{Thesis outline}

The thesis is divided into two parts, with background material in Part I and edited versions of published papers in Part II.

\section{Part I - Background}

The first part introduces the theoretical background for the publications in Part II. Chapter 2 presents basic concepts from graph theory and discusses the use of random networks and network centrality metrics for the development of algorithms. Chapter 3 provides background theory on controllability, with focus on classical notions of controllability that have been recently adapted to the context of complex networks.

\section{Part II - Publications}

The second part consists of edited versions of three publications. Below is a summary of each paper.

\section{Paper A: Controllability of complex networks with unilateral inputs}

Paper $\mathrm{A}$ is an edited version of

Gustav Lindmark and Claudio Altafini. Controllability of complex networks with unilateral inputs. Scientific Reports, 7:1824, 2017a. doi: 10.1038/s41598-017-01846-6.

Summary: In Paper A we study the problem of controlling complex networks with unilateral controls, i.e., controls that can assume only positive or negative values, not both. Given a complex network represented by the adjacency matrix $A$, an algorithm is developed that constructs an input matrix $B$ such that the resulting system $(A, B)$ is controllable with a near minimal number of unilateral control inputs. This is made possible by a reformulation of classical conditions for controllability that casts the minimal unilateral input selection problem into well known optimization problems. We identify network properties that make unilateral controllability relatively easy to achieve as compared to unrestricted controllability. The analysis of the network topology for instance allows us to establish theoretical bounds on the minimal number of controls required. For various categories of random networks as well as for a number of real-world networks these lower bounds are often achieved by our heuristics.

Contribution and background: The author of this thesis contributed with the majority of the work including theoretical derivations, implementations, numerical calculations and the written manuscript. 


\section{Paper B: Minimum energy control for complex networks}

Paper B is an edited version of

Gustav Lindmark and Claudio Altafini. Minimum energy control for complex networks. Scientific Reports, 8(1):3188, 2018a.

Summary: The aim of Paper B is to shed light on the problem of controlling a complex network with minimal control energy. We show first that the control energy depends on the time constants of the modes of the network, and that the closer the eigenvalues are to the imaginary axis of the complex plane, the less energy is required for complete controllability. In the limit case of networks having all purely imaginary eigenvalues (e.g. networks of coupled harmonic oscillators), several constructive algorithms for minimum control energy driver node selection are developed. A general heuristic principle valid for any directed network is also proposed: the overall cost of controlling a network is reduced when the controls are concentrated on the nodes with highest ratio of weighted outdegree vs. indegree.

Contribution and background: The author of this thesis contributed with implementations, analysis and reviewing the manuscript.

\section{Paper C: The role of non-normality for control energy reduction in network controllability problems}

Paper $\mathrm{C}$ is an edited version of

Gustav Lindmark and Claudio Altafini. The role of non-normality for control energy reduction in network controllability problems. arXiv preprint:1806.05932, 2018b.

Summary: Paper C investigates the problem of controlling a complex network with reduced control energy. Two centrality measures are defined, one related to the energy that a control, placed on a node, can exert on the entire network, the other related to the energy that all other nodes exert on a node. We show that by combining these two centrality measures, conflicting control energy requirements, like minimizing the average energy needed to steer the state in any direction and the energy needed for the worst direction, can be simultaneously taken into account. From an algebraic point of view, the node ranking that we obtain from the combination of our centrality measures is related to the non-normality of the adjacency matrix of the graph.

Contribution and background: The author of this thesis contributed with the majority of the work including theoretical derivations, implementations, numerical calculations and the written manuscript.

\subsection{Other publications}

The following additional publications have been authored or co-authored by the author of this thesis: 
Gustav Lindmark and Claudio Altafini. Positive controllability of large-scale networks. In Proceedings of the 2016 European Control Conference (ECC), pages 819-824. IEEE, 2016.

Francesca Ceragioli, Gustav Lindmark, Clas Veibäck, Niklas Wahlström, Martin Lindfors, and Claudio Altafini. A bounded confidence model that preserves the signs of the opinions. In Proceedings of the 2016 European Control Conference (ECC), pages 543-548. IEEE, 2016.

Gustav Lindmark and Claudio Altafini. Topological aspects of controlling large scale networks with unilateral inputs. IFAC-PapersOnLine, 50(1):8315-8320, 2017d.

Gustav Lindmark and Claudio Altafini. Minimum energy control for networks of coupled harmonic oscillators. IFAC-PapersOnLine, 50(1): 8321-8326, 2017c.

Gustav Lindmark and Claudio Altafini. A driver node selection strategy for minimizing the control energy in complex networks. IFACPapersOnLine, 50(1):8309-8314, 2017b. 


\subsection{Contributions}

Below the main contributions of the thesis are listed.

- Exact theoretical bounds on the minimal number of controls required for unilateral controllability based on the network topology. (Paper A)

- An algorithm which identifies a near minimal set of unilateral control inputs that renders a given network controllable. (Paper A)

- We show how the energy required for controlling a network depends on the time constants of the modes of the network. (Paper B)

- Two centrality measures quantifying the importance of the different nodes in controlling a network. Driver node placement based on a combination of these measures results in reduced energy requirements for controlling a network. (Paper C) 



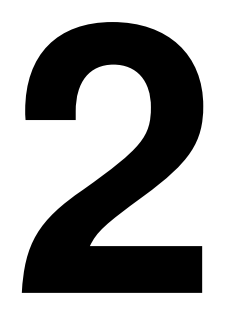

\section{Networks and graphs}

The natural mathematical representation of a network is a graph. In this chapter we present basic concepts from graph theory and discuss the use of random networks and network centrality metrics for the development of algorithms.

\subsection{Graph definitions}

A graph $\mathcal{G}$ is a pair $\mathcal{G}=(\mathcal{V}, \mathcal{E})$ where $\mathcal{V}=\left\{v_{1}, \ldots, v_{n}\right\}$ is a finite set of nodes (or vertices) and $\mathcal{E}$ is a set of edges. An edge $\left(v_{i}, v_{j}\right) \in \mathcal{E}$ connects the nodes $v_{i}, v_{j} \in \mathcal{V}$, the nodes are said to be adjacent to each other and incident to the edge $\left(v_{i}, v_{j}\right)$. In a directed graph, or digraph, the edge $\left(v_{i}, v_{j}\right)$ is directed from $v_{i}$ to $v_{j}$, while in an undirected graph, $\left(v_{i}, v_{j}\right) \in \mathcal{E} \Rightarrow\left(v_{j}, v_{i}\right) \in \mathcal{E}$. A weighted graph has weights associated with each edge.

We say that $\mathcal{G}^{*}=\left(\mathcal{V}^{*}, \mathcal{E}^{*}\right)$ is a subgraph of $\mathcal{G}=(\mathcal{V}, \mathcal{E})$ if $\mathcal{V}^{*} \subset \mathcal{V}, \mathcal{E}^{*} \subset \mathcal{E}$ and the edges in $\mathcal{E}^{*}$ only connect nodes in $\mathcal{V}^{*}$. A path $\mathcal{P}$ in $\mathcal{G}$ is a subgraph of the form $\mathcal{V}^{*}=\left\{v_{i_{1}}, \ldots, v_{i_{j}}\right\}$ and with the edges $\mathcal{E}=\left\{\left(v_{i_{1}}, v_{i_{2}}\right), \ldots,\left(v_{i_{j-1}}, v_{i_{j}}\right)\right\}$. The path is directed from $v_{i_{1}}$ to $v_{i_{j}}$ and the number of edges is the path length. A cycle is a path in which $v_{i_{1}}=v_{i_{j}}$. The simplest form of cycle is a node $v_{i}$ with a self-loop $\left(v_{i}, v_{i}\right)$.

A graph can be specified by its adjacency matrix $A$, i.e. $\mathcal{G}=\mathcal{G}(A)=(\mathcal{V}, \mathcal{E})$. With $|\mathcal{V}|=n$, the matrix $A$ is $n \times n$. When the graph is unweighted, the element on row $j$ and column $i, A_{j i}=1$ if $\left(v_{i}, v_{j}\right) \in \mathcal{E}$ and $A_{j i}=0$ if $\left(v_{i}, v_{j}\right) \notin \mathcal{E}$. In a weighted graph the element $A_{j i}$ specifies the weight of the edge $\left(v_{i}, v_{j}\right)$, and $A_{j i} \neq 0$ if $\left(v_{i}, v_{j}\right) \in \mathcal{E}$.

An undirected graph is connected if there is a path between any two nodes in the graph. The connected components of a graph is its maximal connected subgraphs. Analogously, a directed graph is strongly connected if there is a directed path from each node to every other node, and the strongly connected components 
is its maximal strongly connected subgraphs. The minimal connected/strongly connected component is a single node.

Two distinct nodes that are adjacent (i.e. connected by an edge) are neighbors. For undirected graphs, the degree of a node is its number of incident edges. The degree distribution of a graph is the distribution of the degrees of its nodes. In a directed graph, the in-neighbors of the node $v_{i}$ is given by the set of nodes $\left\{v_{j}, j\right.$ s.t. $\left.\left(v_{j}, v_{i}\right) \in \mathcal{E}\right\}$, and the out-neighbors by the set $\left\{v_{k}, k\right.$ s.t. $\left.\left(v_{i}, v_{k}\right) \in \mathcal{E}\right\}$. The indegree of a node is the number of edges incoming into it, and the outdegree the number of outgoing edges from it. The definitions of indegree and outdegree distributions of a directed graph follow. We say that a node in a directed network is a root if it has indegree zero, and a leaf if it has outdegree zero.

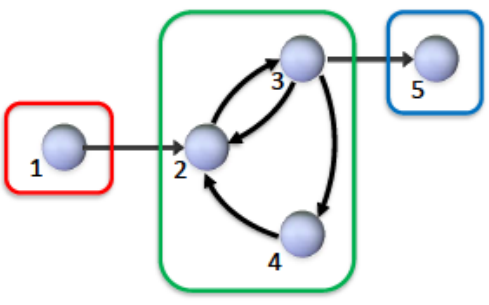

(a)

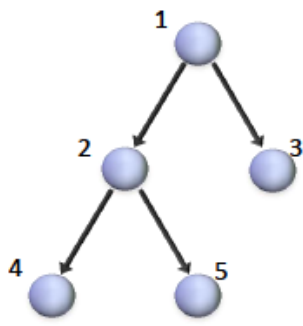

(b)

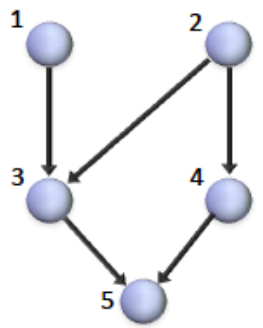

(c)

Figure 2.1: Simple graphs that illustrate key-concepts. (a): The graph has three strongly connected components marked in red/green/blue. The node $v_{1}$ is a root and $v_{5}$ a leaf. The strongly connected component marked in green contains the cycle $\left\{v_{2}, v_{3}, v_{4}, v_{2}\right\}$. In the graph, $v_{2}$ is the node with highest in-degree $\left(k_{\text {in }}=3\right)$ and $v_{3}$ has the highest out-degree $\left(k_{\text {out }}=3\right)$. (b): Rooted directed tree. (c): Directed acyclic graph.

\section{Some special classes of graphs}

A tree is an undirected graph where any two nodes are connected with exactly one path, hence a tree is acyclic. In a directed rooted tree one node is the root and all edges are pointing either away from or towards the root such that there is exactly one path from the root to all other nodes or vice versa. Directed rooted trees belong to the important class of graphs Directed acyclic graphs, DAGs. As the name suggests, a DAG is directed and contain no cycles. In a DAG, there is a topological ordering of the nodes such that $i<j$ for all $\left(v_{i}, v_{j}\right) \in \mathcal{E}$. The topological ordering is unique if and only if there is a directed path containing all the nodes. DAGs are used for instance in computer science to identify dependencies between the execution of different tasks and detect deadlocks, or to model probabilities and causality. The adjacency matrix of a DAG without self-loops is nilpotent.

A graph is said to be complete if there are edges between all pairs of nodes. If the graph is directed then there must be edges in both directions. All off-diagonal entries of the corresponding adjacency matrix are non-zero (the diagonal entries 
are also non-zero if there are self-loops).

\subsection{Random graphs}

It is often interesting to generate random graphs, for instance to evaluate different graph methods or algorithms. By applying a method on a large number of random graphs, statistically-assured conclusions can be drawn about its performance. A random graph is described either by a probability function or a process that generates it. Although random graphs are random, they might have different characteristic properties. One such graph property that often is of interest is the degree of clustering. Clustering is when the neighbors of a node tend to be also neighbors to each other. Another important graph property is the connectivity. A connected graph with high connectivity remains connected even when several nodes or edges are removed from the graph.

The generation of random graphs with specific properties is an active area of research. However, here it is enough to present the two perhaps most common classes of random graphs, Erdős-Rényi networks and scale-free networks. (The terms graph and network are synonymous in this context.)

\section{Erdős-Rényi networks}

In the Erdős-Rényi random network model, the probability that an edge connecting $v_{i}$ and $v_{j}$ exists is $p$ for all $i, j \in 1, \ldots, n$. That is, the probability $p$ is independent of what other edges there are and equal for all pairs of nodes. Denote by $P(k)$ the probability that a node has degree $k$. In an Erdős-Rényi network with $n$ nodes,

$$
P(k)=\left(\begin{array}{c}
n-1 \\
k
\end{array}\right) p^{k}(1-p)^{n-1-k}, k=0,1, \ldots, n,
$$

i.e. the function $P(k)$ is a binomial probability distribution with expected value $n p$ (the nodes have on average $n p$ neighbors). Moreover, the probabilistic degree distribution of Erdős-Rényi networks becomes $n P(k)$. Erdős-Rényi networks are characterized by low clustering but high connectivity (Newman, 2010).

\section{Scale-free networks}

Scale-free networks are networks where the degree distribution follows a power law,

$$
P(k)=C k^{-\gamma}, k=1,2, \ldots, n .
$$

Here, $C$ is a constant and $\gamma$ is the degree exponent of the power law. Several real world networks are claimed to be close to scale-free with $\gamma \in[2,3]$ (Barabási and Albert, 1999). That includes several computer networks like the World Wide Web and collaboration networks. A Scale-free network is for instance the result 
of a preferential attachment process in which the network is constructed incrementally by the addition of new nodes and edges. When an edge is added, the probability that it becomes incident to an existing node $v_{i}$ is higher the higher the degree of $v_{i}$ is. Hence, new nodes are more likely to connect to already "important" nodes. Preferential attachment has been used to model several real world phenomena (Newman, 2010).

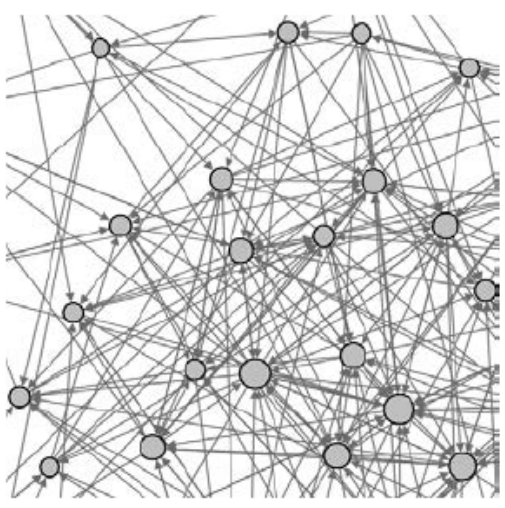

(a)

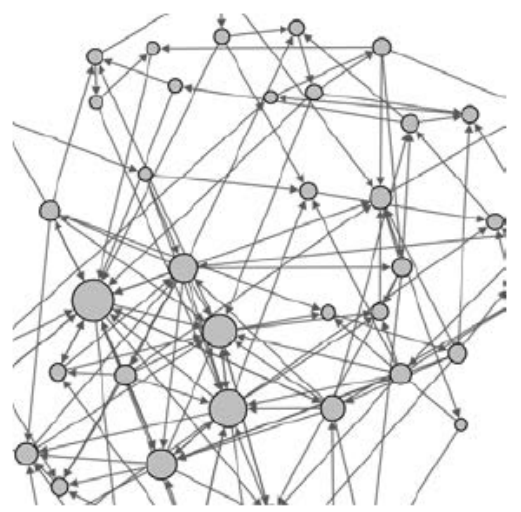

(b)

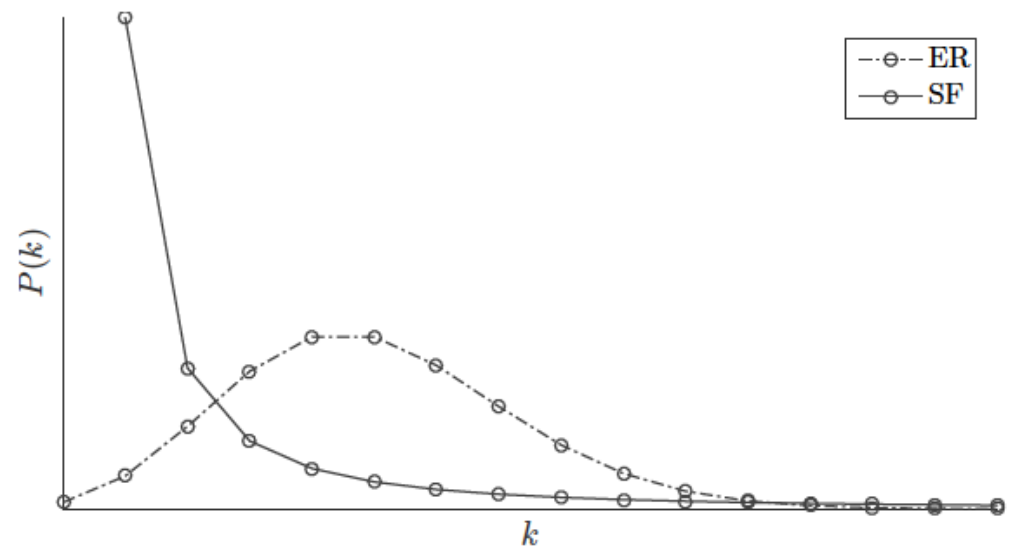

(c)

Figure 2.2: (a): Directed Erdôs-Rényi network. (b): Directed scale free network. In scale-free networks a large fraction of the edges are concentrated around a few hubs while the large majority of the nodes have low degree. (c): The probability distributions $P(k)$ for Erdôs-Rényi and scale-free networks. That is, $P(k)$ is the probability that a node has degree $k$. 


\subsection{Network centrality metrics}

A centrality metric quantifies how important, or central, the different nodes of a network are. There are numerous different centrality metrics and their relevance depends on the type of network and the context in which they are used, see for instance Newman (2010) for an overview. The simplest is probably the degree centrality, which is just the degree of the different nodes. It can be a measure of for instance how influential a person is in a social network or used to study the spread of infection in epidemics. The eigenvector centrality of a node differs from the degree centrality in that the different connections of the node are not equally important. Instead, the centrality of a node is higher if its neighbors have high centrality themselves. Other examples of network centrality metrics are for instance PageRank, known for beeing used by Google Search to rank websites, and the hubness/authority measures used for instance in the HITS algoithm. In particular in a directed network the hub centrality is related to the outgoing edges while the authority centrality is related to the incoming edges.

One way to approach the problem of driver node placement for control of complex networks is to quantify the importance of the different nodes with network centrality measures. There are several centrality metrics proposed for control, see for instance Bof et al. (2017); Liu et al. (2012); Pasqualetti et al. (2014). In paper $\mathrm{C}$ we propose driver node placement based on the combination of two different network centrality metrics. 



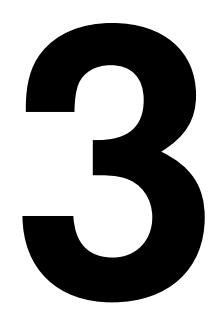

\section{Controllability}

This chapter provides background theory on controllability that is used in the remaining of the thesis. The concept of controllability has a long history, it was first introduced in Kalman (1963) and has has played a fundamental role in system theory since then. While the concept includes a large body of theory and many ramifications (Rugh, 1996; Sontag, 2013), this presentation focuses on classical notions of controllability that have been recently adapted to the context of complex networks. This includes controllability of Linear Time-Invariant (LTI) systems, systems with linear dynamics but constrained control inputs, structural controllability and the interpretation of the reachability Gramian.

\subsection{Controllability of LTI systems}

Consider a continuous-time LTI system

$$
\dot{x}=A x+B u
$$

where $x \in \mathbb{R}^{n}$ is the state vector, $A \in \mathbb{R}^{n \times n}$ is the state update matrix, $B \in \mathbb{R}^{n \times m}$ is the input matrix, and $u$ is the $m$-dimensional input vector. Given the initial state $x(0)=x_{0}$, the solution to the differential equation (3.1) is

$$
x(t)=e^{A t} x_{0}+\int_{0}^{t} e^{A(t-\tau)} B u(\tau) d \tau .
$$

Definition 3.1 (Controllability). The linear state equation (3.1) is called controllable (to the origin) on $\left[0, t_{f}\right]$ if given any initial state $x(0)=x_{0} \in \mathbb{R}^{n}$ there exists an input signal $u(t)$ such that the corresponding solution of 3.1 satisfies $x\left(t_{f}\right)=0$ (Rugh, 1996). 
Closely linked to controllability (to the origin) is the term reachability (or controllability from the origin).

Definition 3.2 (Reachability). The system (3.1) is reachable (or controllable from the origin) in time $t_{f}$ if any $x\left(t_{f}\right)=x_{f} \in \mathbb{R}^{n}$ can be reached from $x(0)=0$ by some control $u(t)$ in time $t_{f}$ (Antsaklis and Michel, 2005).

For continuous-time LTI systems the controllability and reachability properties are equivalent (Rugh, 1996). Furthermore, the time window $\left[0, t_{f}\right]$ can be arbitrarily small when the control inputs are unconstrained.

The assumption that the LTI system (3.1) is controllable (hence also reachable) on $\left[0, t_{f}\right]$ is equivalent to (Zhou et al., 1996)

i) The controllability Gramian

$$
W_{c}\left(t_{f}\right)=\int_{0}^{t_{f}} e^{-A t} B B^{\top} e^{-A^{\top} t} d t
$$

is positive definite.

ii) The reachability Gramian

$$
W_{r}\left(t_{f}\right)=\int_{0}^{t_{f}} e^{A t} B B^{\top} e^{A^{\top} t} d t
$$

is positive definite.

iii) The controllability matrix

$$
\mathcal{C}=\left[\begin{array}{llll}
B A B & \ldots A^{n-1} B
\end{array}\right]
$$

has full row rank.

iv) The matrix $[A-\lambda I B]$ has full row rank for all $\lambda \in \mathbb{C}$.

v) Let $\lambda$ and $x$ be any eigenvalue and any corresponding left eigenvector of $A$, i.e., $x^{\mathrm{H}} A=x^{\mathrm{H}} \lambda$, then $x^{\mathrm{H}} B \neq 0$. Here $x^{\mathrm{H}}$ denotes the complex conjugate transpose of $x$.

vi) The eigenvalues of $A+B F$ can be freely assigned (with the restriction that complex eigenvalues are in conjugate pairs) by a suitable choice of $F$.

Condition iii) is often referred to as the Kalman rank condition, while the conditions iv) and $v$ ) are often called the Popov-Belevitch-Hautus (PBH) tests. The result vi) indicate why the concept of controllability plays a such important role in the classical control theory; the poles of a controllable system can be arbitrarily assigned with state feedback control. 
The controllability Gramian and the reachability Gramian are related by

$$
W_{r}\left(t_{f}\right)=e^{A t_{f}} W_{c}\left(t_{f}\right) e^{A^{\top} t_{f}}
$$

We define the energy of the control input $u(t)$ as

$$
\mathcal{E}(u)=\int_{0}^{t_{f}}\|u(t)\|^{2} d t
$$

The unique control input that steers the network from $x(0)=0$ to $x\left(t_{f}\right)=x_{f}$ with minimum energy is (Rugh, 1996)

$$
\begin{aligned}
& u^{*}(t):=B^{\top} e^{A^{\top}\left(t_{f}-t\right)} W_{r}^{-1}\left(t_{f}\right) x_{f}, \text { and } \\
& \mathcal{E}\left(u^{*}\right)=x_{f}^{\top} W_{r}^{-1}\left(t_{f}\right) x_{f} .
\end{aligned}
$$

For $A$ stable the reachability Gramian converges as $t_{f} \rightarrow \infty$ to the solution of the Lyapunov equation

$$
A W_{r}+W_{r} A+B B^{\top}=0
$$

while for $A$ anti-stable the controllability Gramian converges as $t_{f} \rightarrow \infty$ to the solution of

$$
(-A) W_{c}+W_{c}(-A)+B B^{\top}=0
$$

Remark 3.1. The controllability literature is not always consistent regarding the definitions of the controllability resp. reachability Gramians. What we call the reachability Gramian is sometimes referred to as the controllability Gramian. The distinction is in many cases irrelevant since for continuous LTI systems the concepts are equivalent.

Discrete-time LTI systems: Consider the discrete-time LTI system

$$
x(t+1)=A x(t)+B u(t)
$$

where $x(t) \in \mathbb{R}^{n}$ is the state at time $t \in \mathbb{N}_{0}, A \in \mathbb{R}^{n \times n}, B \in \mathbb{R}^{n \times m}$ and $u(t) \in \mathbb{R}^{m}$. The definitions of controllability and reachability are valid also for this system. There are however some differences as compared to the continuous case that need to be pointed out. For the discrete-time system (3.12), reachability implies controllability, but the reverse is not true. This is related to cases where the $A$ matrix is not invertible. Reachability is normally of greater interest since it is the stronger system quality.

The time-invariant linear state equation is reachable on $\left[0, t_{f}\right], t_{f} \in \mathbb{N}$, if and only if the matrix

$$
\mathcal{C}=\left[B A B \ldots A^{t_{f}-1} B\right]
$$


has full row rank. This is equivalent to that the discrete-time reachability Gramian

$$
W_{r}\left(t_{f}\right)=\sum_{t=0}^{t_{f}-1} A^{t} B B^{\top}\left(A^{\top}\right)^{t}
$$

is positive definite. Unlike the continuous case, reachability can fail due to the size of the time interval $t_{f}$. For $t_{f}>n$ it is enough to consider $\mathcal{C}=\left[B A B \ldots A^{n-1} B\right]$. In analogy with equations (3.7) through (3.9), we define the energy of the discretetime control input $u(t)$ as

$$
\mathcal{E}(u)=\sum_{t=0}^{t_{f}-1}\|u(t)\|^{2} .
$$

The unique control input that steers the network from $x(0)=0$ to $x\left(t_{f}\right)=x_{f}$ with minimum energy is

$$
\begin{aligned}
& u^{*}(t):=B^{\top}\left(A^{\top}\right)^{t_{f}-t-1} W_{r}^{-1}\left(t_{f}\right) x_{f}, \text { and } \\
& \mathcal{E}\left(u^{*}\right)=x_{f}^{\top} W_{r}^{-1}\left(t_{f}\right) x_{f} .
\end{aligned}
$$

For $A$ stable the reachability Gramian converges as $t_{f} \rightarrow \infty$ to the solution of

$$
A W_{r} A^{\top}-W_{r}+B B^{\top}=0
$$

\subsection{Controllability with constrained inputs}

For LTI systems the control inputs are assumed unconstrained, but in many different fields in which controllability of complex networks is studied, the control inputs are intrinsically constrained. Also controllability with constrained inputs has a long history, see Chapter 5 of Jacobson (1977) for a survey. Here we present a key result obtained in Brammer (1972).

Consider the continuous time system (3.1). The admissible controls are all vector functions $u(t)$ taking value in the control restraint set $\Omega$, i.e.

$$
u(t) \in \Omega \subset \mathbb{R}^{m} .
$$

(With unconstrained controls, $\Omega=\mathbb{R}^{m}$.) Brammer's results apply to the weaker controllability notion known as null-controllability.

Definition 3.3 (Null-controllability). The system (3.1) subject to (3.18) is nullcontrollable if there exists an open set $\Gamma \subseteq \mathbb{R}^{n}$ containing the origin for which any $x_{0} \in \Gamma$ can be controlled to the origin in finite time.

Clearly null-controllability is really controllability in a sphere which surrounds the origin and so controllability implies null-controllability but the converse is usually not true. The following conditions are necessary and sufficient for nullcontrollability: 
Theorem 3.1. (Brammer, 1972) Consider the system (3.1) subject to (3.18) satisfying the following conditions:

i) There exists $u \in \Omega$ satisfying $B u=0$ and

ii) the convex hull of $\Omega$ has nonempty interior in $\mathbb{R}^{m}$.

Then the system is null-controllable if and only if

iii) The matrix $\left[\begin{array}{lllll}B & A B & A^{2} B & \ldots & A^{n-1} B\end{array}\right]$ has rank $n$ and

iv) there is no real left eigenvector $v$ of $A$ s.t. $\langle v, B u\rangle \leq 0 \forall u \in \Omega$.

A real eigenvector denotes an eigenvector associated with a real eigenvalue and $\langle v, B u\rangle$ is the Euclidean inner product of the two vectors $v$ and $B u$. An alternative but equivalent formulation of condition $i v$ ) in Theorem 3.1 is:

$\left.i v^{\prime}\right)$ For any real left eigenvector $v$ of $A$ there must be a $u \in \Omega$ such that $\left.\langle v, B u\rangle\right\rangle$ 0 .

It should be pointed out that with constrained control inputs, steering the system in certain directions might rely on the rotation of the unforced system. As a consequence, the time it takes to control the state to the origin can not be chosen arbitrarily.

In Paper A we specialize Brammer's conditions for the case $\Omega=\mathbb{R}_{+}^{m}$, i.e. the control inputs are unilateral.

\subsection{Structural controllability}

Here we present the concept of structural controllability and how it can be used to solve the minimal controllability problem for complex networks. Structural controllability was introduced already in Lin (1974), and the most important theoretical results dates back to the 70s (Glover and Silverman, 1976; Lin, 1974; Shields and Pearson, 1976). However, the concept has regained relevance lately in the context of control of complex networks. A linear time-invariant system with parametrized entries

$$
\dot{x}=A_{\Delta} x+B_{\Delta} u,
$$

is said to be structured if the entries of $A_{\Delta}$ and $B_{\Delta}$ are either fixed zeros or independent parameters.

Definition 3.4 (Structural controllability). The structured system (3.19) is structurally controllable if there exist values of the independent parameters for which the system is controllable (Lin, 1974).

Controllability is a generic property of a structured system, i.e. it is true for almost all values of the independent parameters. More precisely, almost all means all values outside a proper algebraic variety of the parameter space (Murota, 
1987). Hence, the existence of one set of values of the independent parameters for which the system is controllable implies that almost all values of the independent parameters render the system controllable.

Remark 3.2. A structured system is strongly structurally controllable if it is controllable for all values of the independent parameters (Mayeda and Yamada, 1979). However, the cases where strong structural controllability can be determined are quite limited, especially when considering complex networks.

In formulating the conditions for structural controllability it is convenient to introduce the graph $\mathcal{G}\left(A_{\Delta}, B_{\Delta}\right)=(\mathcal{V}, \mathcal{E})$ in which both the states and the inputs are represented with nodes and the edges are given by the non-zero parameters of $A_{\Delta}$ and $B_{\Delta}$,

$$
\begin{aligned}
& \mathcal{V}=\mathcal{V}_{A} \cup \mathcal{V}_{B}, \\
& \mathcal{E}=\mathcal{E}_{A} \cup \mathcal{E}_{B} .
\end{aligned}
$$

Here $\mathcal{V}_{A}=\left\{v_{1}, \ldots, v_{n}\right\}$ is the set of state nodes and $\mathcal{V}_{B}=\left\{v_{n+1}, \ldots, v_{n+m}\right\}$ is the set of input nodes. $\mathcal{E}_{A}=\left\{\left(v_{i}, v_{j}\right) \mid A_{\Delta j i} \neq 0\right\}$ is the set of edges between the state nodes and $\mathcal{E}_{B}=\left\{\left(v_{i}, v_{j}\right) \mid B_{\Delta j i} \neq 0, v_{i} \in \mathcal{V}_{B}, v_{j} \in \mathcal{V}_{A}\right\}$ is the set of edges from the input nodes to the state nodes.

Definition 3.5. A state node $v_{i} \in \mathcal{V}_{A}$ is called inaccessible if and only if there is no directed path reaching $v_{i}$ from any of the input nodes $v_{n+1}, \ldots, v_{n+m}$.

The in-neighborhood set $T(\mathcal{S})$ of a set $\mathcal{S} \subset \mathcal{V}$ is the set of all nodes from which there exist an edge to a node in $\mathcal{S}$, i.e. $T(\mathcal{S})=\left\{v_{j} \mid\left(v_{j}, v_{i}\right) \in \mathcal{E}, v_{i} \in \mathcal{S}\right\} .|\mathcal{S}|$ and $|T(\mathcal{S})|$ are the cardinality of set $\mathcal{S}$ and $T(\mathcal{S})$ respectively.

Definition 3.6. A dilation in the graph $\mathcal{G}=(\mathcal{V}, \mathcal{E})$ is a subset $\mathcal{S} \subset \mathcal{V}$ such that $|T(\mathcal{S})|<|\mathcal{S}|$.

The root nodes are not allowed to belong to $\mathcal{S}$ but may belong to $T(\mathcal{S})$.

Theorem 3.2. (Lin, 1974). The system (3.19) is structurally controllable if and only if

i) The graph $\mathcal{G}\left(A_{\Delta}, B_{\Delta}\right)$ contains no inaccessible state nodes.

ii) The graph $\mathcal{G}\left(A_{\Delta}, B_{\Delta}\right)$ contains no dilation.

The generic rank of the structured matrix $\left[\begin{array}{ll}A_{\Delta} & B_{\Delta}\end{array}\right]$ is defined to be the maximum rank that $\left[\begin{array}{ll}A_{\Delta} & B_{\Delta}\end{array}\right]$ can attain as a function of all the free parameters. The graphical condition ii) of Theorem 3.2 is equivalent to the algebraic condition:

ii') The generic rank of $\left[\begin{array}{ll}A_{\Delta} & B_{\Delta}\end{array}\right]=n$.

In the following, conditions $i$ ) and ii) of Theorem 3.2 will be referred to as the input connected condition and the rank condition. 


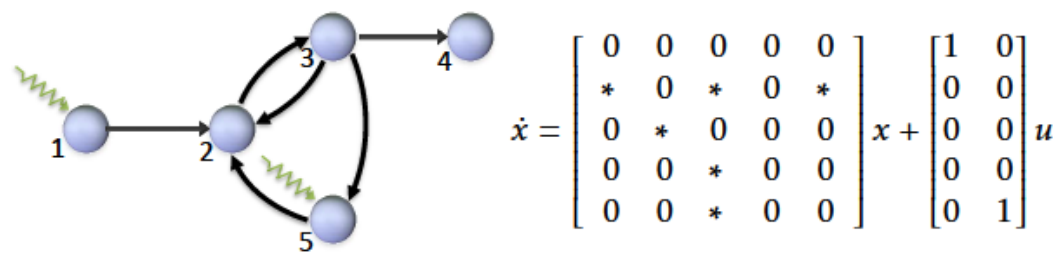

Figure 3.1: The green arrows in the graph indicate control inputs, hence $v_{1}$ and $v_{5}$ are driver nodes. There is a path from the control input applied to $v_{1}$ to all nodes, hence the input connected condition of Theorem 3.2 is met. Furthermore, the set of nodes $\mathcal{S}=\left\{v_{4}, v_{5}\right\}$ with in-neighborhood set $T(\mathcal{S})=$ $\left\{v_{3}\right\}$ is a dilation, and the control input applied to $v_{5}$ is needed in order to meet the rank condition of Theorem 3.2. The generic rank of $\left[A_{\Delta} B_{\Delta}\right]=n$.

\subsection{Minimal controllability of complex networks}

The problem of finding a minimal set of driver nodes that render a given network (structurally) controllable is referred to as the minimal controllability problem (Olshevsky, 2014; Pequito et al., 2014). The structural controllability framework offers efficient graph-based methods to solve this problem when the given network model is linear and structured. The minimal number of driver nodes is an interesting measure for instance when a cost is associated with the construction of each control input. Moreover, it can be considered a measure of the difficulty to control a network. As mentioned in the introduction, a structured network model is a topological model where the location of the edges connecting the nodes is known but not the values of the edge weights. However, results on structural controllability cannot be used for instance for the design of a controller.

For the system (3.19), represented with the graph $\mathcal{G}\left(A_{\Delta}, B_{\Delta}\right)=(\mathcal{V}, \mathcal{E})$, if the generic rank of $A_{\Delta}$ is $\gamma$, then the generic rank of $B_{\Delta}$ must be at least $n-\gamma$ for the rank condition ii) to hold. The number $\mu_{0}=n-\gamma$ is sometimes referred to as the rank deficiency of the network. It is the dimension of the nullspace of $A_{\Delta}$ and also the minimum number of driver nodes that are necessary to meet the rank condition for structural controllability.

The generic rank can be computed using for instance the Dulmage-Mendelsohn (DM) decomposition as in Commault and Dion (2013). By doing that, a number of results follow:

i) The generic rank is obtained, and thereby also the rank deficiency.

ii) It is possible to identify nodes that must be driver nodes.

iii) It is possible to identify nodes such that, if they are added to the set of driver nodes, the generic rank of $\left[A_{\Delta} B_{\Delta}\right]$ is increased by one.

We call a strongly connected component of $\mathcal{G}\left(A_{\Delta}, B_{\Delta}\right)$ that has no incoming edge from any other strongly connected component a Rooted Connected Component (RCC). The input connected condition for structural controllability is met when all RCCs of the graph $\mathcal{G}\left(A_{\Delta}, B_{\Delta}\right)$ are single input nodes. 
By combining the DM-decomposition for the rank condition and other graph algorithms for the identification of RCCs, a method for finding a minimal set of driver nodes in polynomial time is obtained. As will be seen in Paper A, the input connected condition is in many cases met with the driver nodes that are placed to meet the rank condition, meaning that the minimal number of driver nodes becomes $\mu_{0}$, the dimension of the null space of $A_{\Delta}$.

\subsection{Minimum energy control of complex networks}

If $x_{f}$ is a unit-length eigenvector of the Gramian $W_{r}$, then from (3.9) the minimum energy for the state-transition from $x(0)=0$ to $x\left(t_{f}\right)=x_{f}$ equals $x_{f}^{\top} W_{r}^{-1} x_{f}=$ $1 / \lambda$, where $\lambda$ is the eigenvalue associated with $x_{f}$ (Yan et al., 2015).

The control energy can be considered a measure of the effort needed to arbitrarily steer the state, which makes it an interesting cost to minimize. It might also be that a too large control energy cannot be realized by the actuators or that a linear approximation of a nonlinear system becomes invalid when the control inputs are too large. Several approaches have been proposed based on scalar metrics obtained from the control energy, such as

i) The minimal eigenvalue of the reachability Gramian, $\lambda_{\min }\left(W_{r}\right)$ : The energy required to steer the system from the origin along the worst case direction is $1 / \lambda_{\min }\left(W_{r}\right)$.

ii) $\operatorname{Tr}\left(W_{r}^{-1}\right)$ : The trace of the inverse Gramian is proportional to the average energy required to control a system in different directions of the state space.

iii) $\operatorname{Tr}\left(W_{r}\right)$ : The trace of the Gramian is inversely related to the average energy required to control a system, hence when $\operatorname{Tr}\left(W_{r}\right)$ increases the control energy decreases. Note that $W_{r}$ may be singular (and the system not controllable) although $\operatorname{Tr}\left(W_{r}\right)$ is high.

iv) det $W_{r}$ : The volume of the subset of the state space which is reachable from the origin given a fixed amount of control energy is a function of $\operatorname{det} W_{r}$.

See for instance Müller and Weber (1972); Summers et al. (2016) for a thorough description of these and other metrics.

Through the placement of driver nodes, i.e. the design of the $B$ matrix, we can influence the properties of the controllability Gramian, and hence the metrics i) to iv) above. How the controllability Gramian depends on the selected driver nodes is given by Equation 3.4 (or Equation 3.13, discrete time), but to describe how the eigenvalues and eigenvectors of the Gramian depend on the columns of $B$ is not an easy task from that expression.

With only a minimal set of driver nodes for controllability it turns out that for instance $\lambda_{\min }\left(W_{r}\right)$ is often very close to zero, meaning that very large amounts of control energy, sometimes completely unreasonable amounts, are required for control in some direction. More driver nodes are needed to obtain controllability 
in practice, and they should be placed where they contribute the most to the control objectives.

The question "How should a limited number of driver nodes be placed to obtain the lowest possible energy needed for control?" is far from solved although several different approaches have been tried. It is for instance formulated as an optimization problem in Tzoumas et al. (2016) and Summers et al. (2016). Another way to approach the problem is to quantify the importance of the different nodes for controllability with network centrality measures (Bof et al., 2017; Pasqualetti et al., 2014), see also Papers B and C. 



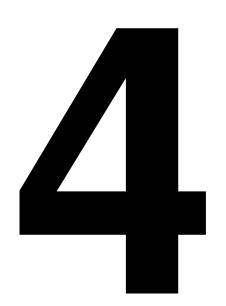

\section{Concluding remarks}

\subsection{Summary of results}

In an effort to render more realistic the problem of controlling complex networks, in the first paper we study the minimal controllability problem given a network model with linear dynamics but unilateral control inputs. The model is motivated by the fact that unilateral controls are more common than bidirectional controls in many contexts. To enable a large scale analysis applicable to complex networks, we cast classical controllability results valid in this particular case into a more computationally-efficient form. The obtained controllability conditions are formulated algebraically in terms of the real eigenspaces of the weighted adjacency matrix $A$ and positively spanning sets. With random edge weight assignments, the non-zero eigenvalues of $A$ are generically simple and the null space is determined by the topology of the network. As a result, the unilateral controllability problem is to a high degree a topological problem. Moreover, we have studied various categories of random networks as well as real-world networks using an algorithm that identifies a near minimal set of unilateral control inputs, and found that two factors essentially determines the minimal unilateral controllability problem: the dimension of the null space of $A$ and to what extent the null space is generated by topological roots or dilations. In contrast, the values of the edge weights have only a minor significance. In comparison, the minimal number of unconstrained control inputs is determined by the dimension of the null space of $A$ but does not depend on whether it is roots or dilations that generate the null space. The difference is considerably large for some classes of networks, almost twice as many unilateral control inputs as unconstrained are needed for networks dominated by hubs with high indegree. However, for most networks the difference is much smaller and for networks dominated by hubs with high outdegree it is negligible. 
The relevance of "theoretical controllability" with its "yes" or "no" answer, has however several shortcomings. On the one hand it can be argued that controllability is an unnecessarily ambitious goal. In many applications it is enough to control a subset of the nodes, i.e. achieve controllability in a subspace of $\mathbb{R}^{n}$. On the other hand, a network that is controllable according to the mathematical definition is often not controllable in practice due to unrealistic requirements on the control energy. This is studied in the Papers B and C, now under the assumption that the control inputs are unconstrained. In Paper B we adopt an LTI network model and allow networks with both stable and unstable eigenmodes. By analyzing numerically how the location of the eigenvalues of the weighted adjacency matrix $A$ influence the reachability Gramian and the energy measures that can be obtained from it, we find that the time constants of the eigenmodes of A play a key role regardless of the number of driver nodes (driver nodes are randomly placed and controllability is ensured). For controllability to the origin, the control inputs must "dominate" the eigenmodes of eigenvalues with positive real part, while for controllability from the origin the eigenmodes of eigenvalues with negative real part are the more difficult to handle. Generally, the energy required for control increases with the magnitudes of the real parts of the eigenvalues. For the special case with purely imaginary spectra we propose three different algorithms for the placement of driver nodes, each one designed for the optimization of one specific energy measure. For the general case with networks with arbitrary eigenvalues, Paper B suggests a strategy for the placement of driver nodes that consists in ranking the nodes by a connectivity property expressed as the ratio between the weighted outdegree and the weighted indegree of the nodes. The strategy outperforms a random placement of equally many driver nodes, and the improvements increases with the connectivity ratio. For instance, the worst-case control energy for directed scale free networks is reduced by several orders of magnitude.

The driver node placement strategy suggested in Paper B based on a numerical analysis is further developed and theoretically investigated in Paper C. A key observation for placement of driver nodes is that what makes a good driver node depends both on its influence over other nodes in the network and on its ability to be controlled indirectly from other nodes. In Paper $\mathrm{C}$ we utilize this and propose the use of two network centrality measures, denoted $p$ and $q$, for the placement of driver nodes. They are both based on energy flow considerations: $p_{i}$ is the energy flow from node $v_{i}$ to all nodes in the network and $q_{i}$ is the energy flow from all nodes to $v_{i}$. The centrality $p$ reflects the network impact of a node, in fact, a driver node placement based on $p$ optimizes the trace of the reachability Gramian control energy metric. The measure $q$ instead quantifies the ability to control a node indirectly from the other nodes. It is in a sense an extension of the rank condition for structural controllability, which requires nodes that cannot be indirectly controlled to be driver nodes themselves. Low $q_{i}$ corresponds to nodes that "almost" cannot be controlled indirectly. Moreover, the distribution of $q$ for the nodes in a network gives a lower bound on the worst case control energy. The centralities $p$ and $q$ are combined into node rankings where nodes with high $p$ and low $q$ are preferred. Algebraically, the rankings relate to the non- 
normality of the $A$ matrix: the driver node placements based on them exploits the non-normality of $A$ and results in reduced energy requirements for controlling the network, i.e. both the average energy needed to steer the state in any direction and the energy needed for the worst direction are simultaneously improved w.r.t. a random driver node placement. As with the ranking suggested in Paper B, to which the current rankings are closely related, the improvements are visible but limited for Erdős-Rényi networks, but significantly higher, 3-4 orders of magnitude, for the more non-normal directed scale-free networks.

\subsection{Future work}

An interesting extension of our unilateral controllability analysis would be to also include metrics for the "practical degree of controllability". Metrics based on the reachability Gramian are motivated by the unique control input that steers a network from one point in the state-space to another with minimal energy. But for arbitrary states, such control input assumes both positive and negative values whereas the reachability Gramian cannot be used for unilateral controllability.

From Paper B, obviously, the eigenvalues of $A$ are important for the control energy. With this in mind, an interesting question that relates to both Paper $\mathrm{B}$ and Paper $\mathrm{C}$ is if also the non-normality of $A$ can be formally linked to the difficulty to control a network. Furthermore, whether or not a node is suitable as a driver node depends not only on individual properties like the ones identified in Paper C. Some measures of the control energy improves when the driver nodes complements each other. Hence, the placement of driver nodes is a combinatorial problem that cannot be solved with only scalar network centrality metrics. 



\section{Bibliography}

P.J. Antsaklis and A.N. Michel. Linear Systems. Birkhäuser Boston, 2005.

Albert-László Barabási and Réka Albert. Emergence of scaling in random networks. Science, 286(5439):509-512, 1999.

Nicoletta Bof, Giacomo Baggio, and Sandro Zampieri. On the role of network centrality in the controllability of complex networks. IEEE Transactions on Control of Network Systems, 4(3):643-653, 2017.

Robert F. Brammer. Controllability in linear autonomous systems with positive controllers. SIAM Journal on Control, 10(2):339-353, 1972.

Francesca Ceragioli, Gustav Lindmark, Clas Veibäck, Niklas Wahlström, Martin Lindfors, and Claudio Altafini. A bounded confidence model that preserves the signs of the opinions. In Proceedings of the 2016 European Control Conference (ECC), pages 543-548. IEEE, 2016.

Yu-Zhong Chen, Le-Zhi Wang, Wen-Xu Wang, and Ying-Cheng Lai. Energy scaling and reduction in controlling complex networks. Royal Society Open Science, 3(4), 2016. doi: 10.1098/rsos.160064.

Christian Commault and Jean-Michel Dion. Input addition and leader selection for the controllability of graph-based systems. Automatica, 49(11):3322 - 3328, 2013. ISSN 0005-1098.

K. Glover and L. Silverman. Characterization of structural controllability. IEEE Transactions on Automatic control, 21(4):534-537, 1976.

D.H. Jacobson. Extensions of Linear-Quadratic Control, Optimization and Matrix Theory, volume 133 of Mathematics in Science and Engineering. Academic Press, London, 1977.

Rudolf Emil Kalman. Mathematical description of linear dynamical systems. Journal of the Society for Industrial \& Applied Mathematics, Series A: Control, 1(2):152-192, 1963. 
Guoqi Li, Wuhua Hu, Gaoxi Xiao, Lei Deng, Pei Tang, Jing Pei, and Luping Shi. Minimum-cost control of complex networks. New Journal of Physics, 18(1): $013012,2016$.

Ching Tai Lin. Structural controllability. IEEE Transactions on Automatic Control, 19(3):201-208, 1974.

Gustav Lindmark and Claudio Altafini. Positive controllability of large-scale networks. In Proceedings of the 2016 European Control Conference (ECC), pages 819-824. IEEE, 2016.

Gustav Lindmark and Claudio Altafini. Controllability of complex networks with unilateral inputs. Scientific Reports, 7:1824, 2017a. doi: 10.1038/ s41598-017-01846-6.

Gustav Lindmark and Claudio Altafini. A driver node selection strategy for minimizing the control energy in complex networks. IFAC-PapersOnLine, 50(1): 8309-8314, 2017b.

Gustav Lindmark and Claudio Altafini. Minimum energy control for networks of coupled harmonic oscillators. IFAC-PapersOnLine, 50(1):8321-8326, 2017c.

Gustav Lindmark and Claudio Altafini. Topological aspects of controlling large scale networks with unilateral inputs. IFAC-PapersOnLine, 50(1):8315-8320, $2017 d$.

Gustav Lindmark and Claudio Altafini. Minimum energy control for complex networks. Scientific Reports, 8(1):3188, 2018 a.

Gustav Lindmark and Claudio Altafini. The role of non-normality for control energy reduction in network controllability problems. arXiv preprint:1806.05932, 2018b.

Yang-Yu Liu, Jean-Jacques Slotine, and Albert-László Barabási. Control centrality and hierarchical structure in complex networks. Plos one, 7(9):e44459, 2012.

Charles P. Madenjian et al. Dynamics of the lake michigan food web, 1970-2000. Canadian Journal of Fisheries and Aquatic Sciences, 59(4):736-753, 2002.

Iven Mareels, Erik Weyer, Su Ki Ooi, Michael Cantoni, Yuping Li, and Girish Nair. Systems engineering for irrigation systems: Successes and challenges. Annual reviews in control, 29(2):191-204, 2005.

Hirokazu Mayeda and Takashi Yamada. Strong structural controllability. SIAM Journal on Control and Optimization, 17(1):123-138, 1979.

P.C. Müller and H.I. Weber. Analysis and optimization of certain qualities of controllability and observability for linear dynamical systems. Automatica, 8(3):237 - 246, 1972. ISSN 0005-1098. doi: http://dx.doi.org/10.1016/ 0005-1098(72)90044-1. 
Kazuo Murota. Systems analysis by graphs and matroids. In Algorithms and Combinatorics, volume 3. Springer Verlag Berlin, 1987.

Jose C. Nacher and Tatsuya Akutsu. Analysis of critical and redundant nodes in controlling directed and undirected complex networks using dominating sets. Journal of Complex Networks, 2(4):394-412, 2014. doi: 10.1093/comnet/ cnu029.

Mark Newman. Networks: an introduction. Oxford university press, 2010.

A. Olshevsky. Minimal controllability problems. IEEE Transactions on Control of Network Systems, 1(3):249-258, Sept 2014. ISSN 2325-5870. doi: 10.1109/ TCNS.2014.2337974.

Alex Olshevsky. Eigenvalue clustering, control energy, and logarithmic capacity. Systems \& Control Letters, 96:45-50, 2016.

Fabio Pasqualetti, Sandro Zampieri, and Francesco Bullo. Controllability metrics, limitations and algorithms for complex networks. IEEE Transactions on Control of Network Systems, 1(1):40-52, 2014.

Sergio Pequito, Guilherme Ramos, Soummya Kar, A. Pedro Aguiar, and Jaime Ramos. On the exact solution of the minimal controllability problem. arXiv preprint:1401.4209, 2014.

Wilson J. Rugh. Linear system theory. Prentice-Hall, Inc., second edition, 1996.

Robert Shields and J. Pearson. Structural controllability of multiinput linear systems. IEEE Transactions on Automatic control, 21(2):203-212, 1976.

Eduardo D. Sontag. Mathematical control theory: deterministic finite dimensional systems, volume 6. Springer Science \& Business Media, 2013.

Tyler H. Summers, Fabrizio L. Cortesi, and John Lygeros. On submodularity and controllability in complex dynamical networks. IEEE Transactions on Control of Network Systems, 3(1):91-101, 2016.

Girma S. Tewolde. Sensor and network technology for intelligent transportation systems. In Electro/Information Technology (EIT), 2012 IEEE International Conference on, pages 1-7. IEEE, 2012.

Núria Ballber Torres and Claudio Altafini. Drug combinatorics and side effect estimation on the signed human drug-target network. BMC Systems Biology, 10(1):74, Aug 2016. ISSN 1752-0509. doi: 10.1186/s12918-016-0326-8. URL https://doi.org/10.1186/s12918-016-0326-8.

Vasileios Tzoumas, Mohammad Amin Rahimian, George J. Pappas, and Ali Jadbabaie. Minimal actuator placement with bounds on control effort. IEEE Transactions on Control of Network Systems, 3(1):67-78, 2016. 
Gang Yan, Jie Ren, Ying-Cheng Lai, Choy-Heng Lai, and Baowen Li. Controlling complex networks: How much energy is needed? Physical Review Letters, 108: 218703, May 2012. doi: 10.1103/PhysRevLett.108.218703.

Gang Yan, Georgios Tsekenis, Baruch Barzel, Jean-Jacques Slotine, Yang-Yu Liu, and Albert-László Barabási. Spectrum of controlling and observing complex networks. Nature Physics, 11(9):779-786, 2015.

Kemin Zhou, John Comstock Doyle, Keith Glover, et al. Robust and optimal control, volume 40. Prentice hall New Jersey, 1996. 


\section{Papers}

The papers associated with this thesis have been removed for copyright reasons. For more details about these see:

http://urn.kb.se/resolve?urn=urn:nbn:se:liu:diva-150886 